\title{
Designing a wearable device for step analyzing
}

\author{
Joaquín Pineda-Gutiérrez, Lourdes Miró-Amarante ${ }^{1}$, Mariló Hernández-Velázquez ${ }^{2}$, Francisco Sivianes-Castillo ${ }^{2}$ \\ and Manuel Domínguez-Morales ${ }^{1}$
}

\begin{abstract}
During the locomotion, the foot is a contact surface and a source of interaction with the external environment. Because of this, its health is important to monitor in order to precociously diagnose its problems. General or specific pathologies can change its physiological function. The measurements of pressure in both dynamic and static conditions can avoid, and even prevent, a worsening of a foot or inferior limb lesion. Plantar pressure exams are then essential to create personalized soles. Foot plantar pressure is the pressure field that acts between the foot and the support surface during everyday locomotive activities. In this work, a plantar pressure measurement system was developed to identify areas with higher or lower pressure load to allow the creation of a personalized sole. The designed device has been planned to be attached to the patient ankle allowing maximum comfort during the normal gait. A series of resistive sensors is attached to the shoe sole, which is then connected to a device placed around the ankle, measures the pressure variations in static condition or during the gait cycle. The measured data are transmitted via Bluetooth to the correlated software. The software of the measurement system shows the pressure in each small sensor element of the sole in real time and saves the data in a file.
\end{abstract}

\section{INTRODUCTION}

Nowadays it is very common to find people who have problems in the ankles, knees or hip derived from the way of walking. Focusing on it, a person can be pronator, supinator or neutral depending on his way of stepping. A pronator person uses the inner part of the sole of the foot to a greater extent when it comes to propelling, while a supinator uses the outer part of the foot more.

Among the problems we can have if we do not walk correctly, we can find some such as Fractures, Ankle Sprain, Pimple Pain or Plantar Fasciitis [7].

To avoid these problems, a podiatrist (or physiotherapist) performs a walking study of the patient's footprint, in order to detect these problems and indicate exercises to correct them.

The classic walking study consists of walking in a straight line through a sensorized surface that emulates a path of several meters length. This surface records the information of each step to analyze it later.

The main problem with this analysis mechanism is the psychological component: the patient knows that he is being analyzed and tries to walk in the best possible way. With this, in many cases the information received does not correspond to a $100 \%$ with the patient's way of walking.

\footnotetext{
${ }^{1}$ Architecture and Computer Technology Department, University of Seville, SPAIN. mdominguez@atc.us.es

${ }^{2}$ Electronic Technology Department, University of Seville, SPAIN.
}

In order to avoid this psychological component, the device that is detailed in this article arises.

This work is based on an instrumented footwear insole [1]. these devices have multiple applications such as in the orthopedic field, for the study of foot function; in the orthoprosthetic field, for the design of footwear, insoles and prostheses; in the biomechanical field, for the study of normal gait or pathological; in sports medicine, for the study of the most appropriate athletic footwear in each athletic modality and taking into account the characteristics of the footprint and the foot.

The instrumented footwear insoles are adjusted to the biomechanical anatomy of the foot [2][8], in this way, they allow to have an objective method to analyze the walking way without conditioning the step and support, with freedom of movement and without space limitation. The pressure measurement system has the advantage that it allows the measurement of both dynamic and static pressure, in any environment and terrain. Given its design and dimensions, it is a portable system; the number of sensors is considerably low, those necessary to support the weight of the patient; and give accuracy in the measurement, which is also one of the most economical options.

The rest of the paper is divided in the following way: first, the footstep phases are analyzed. Subsequently, the implemented device is presented. Next, the algorithms used to filter the information and send it are exposed. Next, the user application for the analysis of the information received is shown. And, finally, the conclusions are presented.

\section{FOOTSTEP PHASES}

\section{A. Analyzing the walking process}

During the march, three distinct phases are distinguished according to the area of the foot involved [3]:

- Impact: first contact zone of the foot with the ground. It is made with the heel and supports a great tension at the moment of impact.

- Support: the second phase. In it the foot is completely supported on the floor and the weight of the person is distributed between the heel and the forefoot through the bridge.

- Drive: last phase. Here, the foot only presents the front part in contact with the ground, and it is when it pulls with the ground to start the movement again.

During the march, these three phases are constantly repeated alternately between the two feet, while one 
of them is in phase 3 , the other is in phase 1 and vice versa [5]. And, during the second phase, one of the feet is fully supported on the ground while the other is in the air.

However, this cycle only remains constant if the person is only walking. While we run, or sprint, there is a moment in which both feet are in the air, this happens after the phase, where the other foot, instead of leaning, stays in the air until the first one has left of having contact with the ground, it is then when phase 1 of the second begins [6][12].

\section{B. Types of sensors}

Pressure sensors are used to perform a biomechanical study of the different parameters that characterize the tread [4]. Pressure sensors, that obtain the force values exerted during the tread, provide information about the maximum pressure point, the impulse, the load forces, the dimensions, the support surface, the speed of movement, the percentages of contact, the angles and the axes of the foot. In the static phase, record the image of the footprint, define the location of the barycenter, as well as the maximum pressure points of each limb and the distribution of loads between the forefoot and the hindfoot. And, in the dynamic phase visualize the consecutive record according to the time, of all the data relative to the foot during the development of the normal step. During the dynamic phase it is also possible to record the area covered between each step, the maximum pressure exerted in each individual phase during the development of the step, the speed of movement of the foot in each phase and the force exerted by the weight during the phases of support and impulse. This allows us to perform a procedure that measures the distribution of plantar pressures in the erect position, either in static or dynamic phase, providing the values of the pressures in colors, the point of maximum pressure, the support surface and the corporal barycenter or the projection of the center of gravity to the footprint.

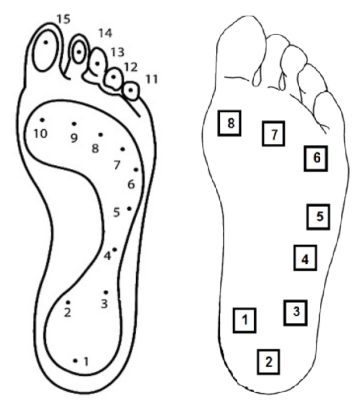

Fig. 1. (left) Foot pressure points, (right) Sensors location

\section{Sensors location}

To select the best position to place the sensors, the anatomy of the foot, the diseases and the types of footprints (pronator, supinator, neutral) are taken into account. According to [1], the sole can be divided into 15 areas: the heel (areas 1-3), the arch (areas 4-5), the metatarsus (areas 6-10) and the fingers (areas 11-15) as shown in Fig. 1-left.

The measurement of the force in these positions can be used to derive information about the structure and function of the inferior artisans and of the whole body in general. In the biomechanics of the march, the walking cycle is defined as the sequence of events that takes place between two successive repetitions of the contact of the same heel with the ground. It is divided into three phases for each leg: In the impact phase, the part that comes into contact with the ground is the part of the heel. So in this it can be useful to place two / three sensors in such a way that they show: a similar value (neutral), the internal sensor has the highest value (Pronation), the external sensor has the highest value (Supination). In the support phase, the foot is in full contact with the ground, both the heel and the front. The weight of the body is distributed between these two zones through the bridge of the foot. To have a better view of the footprint, one or two sensors can be placed in the part of the foot arch to quickly and easily detect a supinatory walk. In the pushing phase only the forefoot is in contact with the ground. With the gauges we have placed in front is enough to know the type of footprint, it is not necessary to place any in the area of the toes [14].

In conclusion, 6 positions are chosen between the heel (numbered with 1,2 and 3 in Fig. 1-left) and the metatarsal (numbered with 6,7 and 8) because these areas have a higher pressure during the activity normal of a person. But if we want to have a more precise study we can put one / two more sensors (numbered with 4 and 5) in the arch of the foot.

\section{EMBEDDED SYSTEM}

The implementation has been carried out over an embedded system [10]. The system is composed of a microcontroller, the FSR force sensors, a bluetooth modem and a battery (among some basic electronic components).

\section{A. Components}

The components are listed and described:

- Microcontroller: ATMega328 on a Arduino Nano platform. It has eight analog inputs (we need seven for the sensors).

- FSR force sensors: resistive sensors whose resistance varies depending on the force exerted on them. Because they are passive sensors, they must be mounted on a resistive divider in order to measure the voltage drop.

- Bluetooth: wireless modem controlled as a virtual serial port that allows the sending of information encoded as strings of characters. 


\section{B. System assembly}

To connect FSR sensors to the microcontroller, a prototyping PCB has beed designed and soldered. It includes the connections for the micorcontroller board, the bluetooth modem, the FSR sensors and some buttons and leds to control the device. FSR sensors have been placed in the footwear insole and connected to the PCB. Finally, to power the system, a powerbank has been used. You can see Fig. 2 to appreciate the final result.

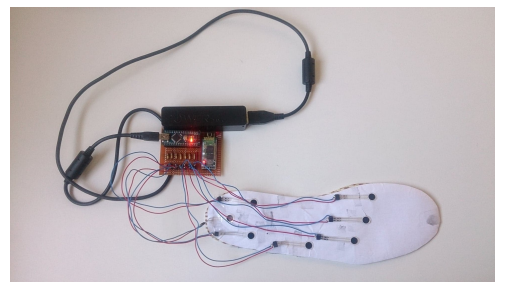

Fig. 2. Instrumented footwear insole

\section{FILTERING AND PROCESSING ALGORITHMS}

In the firmware, we capture the data from the sensors, filter it and use the Bluetooh device to transfer it.

\section{A. Capturing and analyzing data}

The system captures the FSR's information through the microcontroller's "Analog-to-Digital Converter" (ADC) and transformed into voltage range. As the importance of the system resides on the difference between the information received by the FSR sensors, there is not need to convert that to a force value.

In order to eliminate the noise from the information received, we need to filter this data [9] [11]: the sensors' values are stored in an array whose size depends on the sampling speed and the communication rate. Tests have been done with a variable size between 10 and 20 . We will need as many arrays as FSR sensors we have. The filtering process is descibed:

1) The average value for each array is calculated.

2) We define a second array for each FSR sensor, which contains the variances.

3) We calculate the average of the variances array: "standard deviation".

4) To determine the outliers values, we define the limits of the range: established by the average value plus (and less) the standard deviation. Values placed outside this range are considered outliers and are eliminated.

5) Once the noise is eliminated, the average value is calculated again: final force value.

The above procedure is applied for each of the available sensors. The final average values obtained from the cleaned arrays are the values used to be sent by bluetooth to the computer for later analysis.

\section{B. Wireless communication}

A serial port library is used to control the Bluetooth device. This library creates a virtual communication port initialized at a concrete baudrate value of $9600 \mathrm{bits} / \mathrm{sec}$. Each time the data is processed and filtered, the final values are sent using the bluetooth communication. The frame sent starts with a control character ('[') and ends with another (']'). Between them, FSR sensors information are placed (separated between them using ','). This arrangement allows to control the information received: if the device does not receive the start and end control characters, it does not process the frame; and, in addition, if it does not receive all FSR sensors values, it does not process it either.

\section{USER APPLICATION}

Through the visualization system we will show the data captured by the sensors in a computer (in the future, this will be changed for a smart phone)

The visualization system must be accessible to all users regardless of the level of qualification. For that reason, it has been thought of simple interfaces, easy to use, pleasant and flexible. The programming language used will be $C \#$, within the Microsoft Visual Studio platform.

The full version of the software will consist of four windows that we will describe briefly in the following sections:

- Patient Identification window.

- New Patient Window.

- Summary window.

- Registered Patient Window.

\section{A. Patient Identification Window}

It will allow us to search the patient within the Patient Folder: using name, surname and zip code. If the patient is not in the database, a patient message not found will appear. In addition, the window presents another button to add a new patient: This opens the New Patient window.

\section{B. New Patient Window}

It presents the different fields that are necessary to fill in to register the patient in the database. Once the fields have been filled out, a folder is created automatically within the Patient Folder. All files of each exam will be in this folder.

\section{Summary window}

It shows the main data of the patient (Name, Surname, Zip Code, Email, etc.) to confirm that the patient found is the one that was sought to proceed with the examination. In this way, the "Back" key allows you to return to the previous page and the "Continue" button to continue to the acquisition window. 


\section{Registered Patient Window}

It shows an interface where the arrangement of the sensors on the drawing of a standing plant appears on the screen. The acquired value will vary in a range of colors where dark blue indicates absence of pressure and red maximum pressure. This windows has different sections:

- Readings related to the last 10 steps of the patient.

- A text area to insert comments from the user.

- A section dedicated to the buttons: for commands "back", "start", "finish" and "save".

\section{Tests AND CONCLUSSiOnS}

A baropodometric exam consists on two phases [13]:

- In static, the patient is positioned on a platform to detect plantar pressures in the erected position.

- In dynamics, the patient develops a series of steps evaluating the pressures and how the feet develop the phases of the step.

An exclusively static study on the platform is insufficient considering that certain anomalies may not be present in this type of examination, but only be surveyed along the way.

Nowadays, in a baropodometric examination, dynamic analysis is performed by walking the patient on a platform where it is essential that only one foot rests on it while the other remains outside, forcing the patient to develop a pre-established path. Therefore, it is believed that the presence of the platform influences patients, who pay particular attention to the execution of the step, often slowing down the speed of the path and introducing a movement of the unnatural lower limb in proximity to the platform.

The device created, which allows however also a static examination, is mainly aimed at a dynamic analysis, since it can be conveniently inserted into the patient's footwear, allowing him to walk more smoothly and reproduce more faithfully his standard step. To assess the performance has been tested with three types of footprint different: neutral, prone and supine.

For a neutral support, the results obtained indicate that in the initial contact phase, when the subject starts the step supporting the heel on the ground, the support is neutral (sensors 1, 2 and 3 from Fig. 1-left) and also in propulsion / impulse dominates the neutral support (sensors number 6 and 7 from Fig. 1-left).

In a supine support (see Fig. 3), most of the weight of the body is distributed in the external zone of the foot, subjecting it to greater tension and effort and causing its inclination toward the outside. In a prone support, the weight of the body is distributed in the inner part of the foot, therefore the sensors located in this part notice higher pressure values.

We conclude that we have designed and manufactured a system integrated in a shoe to detect the type of footprint of a person. The system consists of a series of

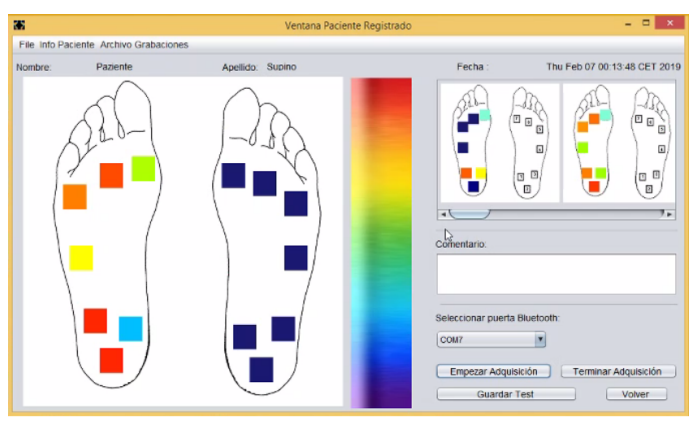

Fig. 3. Supine patient

seven resistive force sensors integrated in the footplate of the shoe and of the device attached to the patient's ankle connected by a Bluetooth module to the data visualization software installed on the computer. The system has proven to be able to capture in real time the information of the patient's footprint and, thanks to the filtering process, the received information is reliable.

\section{REFERENCES}

[1] L. Shu et al., In-shoe plantar pressure measurement and analysis system based on fabric pressure sensing array, Information Technology in Biomedicine, IEEE Transactions on 14 (2010), no. 3, 767775 .

[2] Bamberg, S. et al. (2008). Gait analysis using a shoe-integrated wireless sensor system. IEEE transactions on information technology in biomedicine, 12(4), 413-423.

[3] Kidder, S. et al. (1996). A system for the analysis of foot and ankle kinematics during gait. IEEE Transactions on Rehabilitation Engineering, 4(1), 25-32.

[4] Giacomozzi, C. (2010). Hardware performance assessment recommendations and tools for baropodometric sensor systems. Annali dell'Istituto superiore di sanita, 46, 158-167.

[5] Davis III, R. et al. (1991). A gait analysis data collection and reduction technique. Human movement science, 10(5), 575-587.

[6] Whittle, M. W. (1993). Gait analysis. In The Soft Tissues (pp. 187-199).

[7] Perry, J. and Davids, J. R. (1992). Gait analysis: normal and pathological function. Journal of Pediatric Orthopaedics, 12(6), 815.

[8] Morris, S. J. and Paradiso, J. A. (2002). Shoe-integrated sensor system for wireless gait analysis and real-time feedback. In Engineering in Medicine and Biology, 2002. 24th Annual Conference and the Annual Fall Meeting of the Biomedical Engineering Society EMBS/BMES Conference, 2002. Proceedings of the Second Joint (Vol. 3, pp. 2468-2469). IEEE.

[9] Carson, M. et al. (2001). Kinematic analysis of a multi-segment foot model for research and clinical applications: a repeatability analysis. Journal of biomechanics, 34(10), 1299-1307.

[10] Tao, W. et al. (2012). Gait analysis using wearable sensors. Sensors, 12(2), 2255-2283.

[11] Katoh, Y. et al. (1983). Biomechanical analysis of foot function during gait and clinical applications. Clinical orthopaedics and related research, (177), 23-33.

[12] Lee, L. et al. (2002). Gait analysis for recognition and classification. In Automatic Face and Gesture Recognition, 2002. Proceedings. Fifth IEEE International Conference on (pp. 155162). IEEE.

[13] Robain, G. et al. (2006). A baropodometric parameter to analyze the gait of hemiparetic patients: the path of center of pressure. In Annales de readaptation et de medecine physique (Vol. 49, No. 8, pp. 609-613).

[14] Descatoire, A. et al. (2009). Baropodometric information return device for foot unloading. Medical engineering and physics, 31(5), 607-613. 